\title{
Modeling and simulation of propulsion system for electronically con- trolled marine diesel engine
}

\author{
Yuanqing Wang ${ }^{1}$,Qi Wang ${ }^{1}$, Hongyu Qiao ${ }^{1}$,Juan Su ${ }^{1 *}$,Liang An ${ }^{1}$
}

(1 Marine Engineering Department, Nantong Shipping College, Nantong 226010, Jiangsu,

China)

Keywords: electronically controlled; diesel; propulsion system; modeling; simulation

Abstract: In order to analyze electronically controlled marine diesel engine performance and design reliable control algorithm, According to SULZER 7RT-flex60C type electronic control diesel engine, established marine electronically control diesel propulsion system simulation model by mean value model. Because of increased common rail of electronically control diesel engine, the traditional average model is improved. For the fuel common rail system modeling, design rail pressure control strategy, realize the rail pressure and fuel injection control. then carry out the simulation experiment, the experimental results show that the simulation model can better simulate electronic control diesel engine propulsion system dynamic performance and interaction mechanism. This model can be used for marine electronically control diesel propulsion system performance analysis and algorithm design.

\section{Introduction}

Because of more economical and environmental advantages, electronically controlled diesel engine is widely to the shipping market. The key to realize energy saving and emission reduction is that the added electronically controlled common rail system which improved the flexible control of diesel engine, so that the adverse impact reduced to much low levels about engine speed and load on fuel consumption and exhaust emission. Electronically controlled common rail system and its control strategy were the focus of research on electronic controlled diesel engine. To analyze the electronically controlled diesel engine performance, the key is designing a stable and reliable method to control and establish a reliable model of electronic control diesel propulsion system. In the ship propulsion system, diesel engine model is the core. Diesel engine model mainly include: the transfer function model, the mean value model and the quasi steady state model. The model accuracy is enhanced in turn, but the calculation is more complicated, time-consuming longer. A quasi-dimensional model of the Mean value model parameters input is less and the model precision is higher than the transfer function model that can correctly describe the performance of the diesel engine ${ }^{[1-3]}$. So we chose the mean value model.

Wartsila RT - flex series is a representative of the electronic control diesel engine. According to SULZER 7RT-flex60C type electronic control diesel engine, established marine electronically control diesel propulsion system simulation mode. Compared with the traditional diesel engine

(WECS9500) 7RT-flex60C Electronically Controlled Diesel canceled cam control and added the common-rail unit and electronic control system ${ }^{[4]}$. Therefore, the mean value model of machine diesel engine in this article is improved. The high pressure common rail fuel injection system adopted the way of empirical formula, combined with look-up table modeling, designed rail

\footnotetext{
*Project supported by the National Natural Science Foundation of China (No. 51179102).

*Corresponding Author.

Email address: wangyqdmu@ 163.com (yuanqing wang).
} 
pressure control strategy and achieved fuel injection control. The propeller characteristic is calculated with regression algorithm. The speed of ship is calculated with equations of motion in the direction of navigation ${ }^{[5]}$. Then integrated with ship, diesel engine and propeller models, form a whole system of electronic control diesel propulsion system model. On the basis, analysis of dynamic characteristics of propulsion system and the interaction mechanism through simulation experiments of propulsion system with dynamic behavior.

\section{Electronic controlled diesel engine propulsion system model}

\section{The principle of propulsion system}

According to Newton's second law, the ship velocity can be calculated by the following formula

$$
M \frac{d V_{s}}{d t}=M_{e}-R_{s}=(1-\mathrm{h}) \mathrm{M}_{p}-R_{s}
$$

In Eq. (1), $M$ denotes the sum of quality with ship and hull entrained water quality; $V_{s}$ denotes the speed of the ship; Respectively, $\mathrm{M}_{p}, M_{e}$ and $R_{s}$ denote the thrust of the propeller, the effective thrust of the propeller and the resistance of the ship.

When the propeller and the diesel engine is connected, according to the law of moment of momentum, the engine speed is calculated by the following formula

$$
\frac{d n_{e}}{d t}=\frac{T_{\mathrm{d}}-T_{p}}{I} \frac{60}{2 \pi}=\frac{=T_{\mathrm{i}}-T_{f}-T_{p}}{I} \frac{60}{2 \pi}
$$

In Eq. (2), $I$ denotes the total moment of inertia with diesel engine, shaft and propeller blades; $T_{\mathrm{d}}$ denotes output torque of the diesel engine; $T_{\mathrm{i}}$ denotes the average the diesel engine indicated torque; $T_{f}$ denotes the average friction torque of diesel engine; $T_{p}$ denotes the load torque of diesel engine.

\section{The model of diesel engine system}

Mean value model is combined with the volumetric model and quasi steady state model. Similar to the volumetric method, the diesel engine control unit is divided into a series of volumes, including electronically controlled common rail unit, diesel body, exhaust pipes, turbines, compressors, coolers and scavenging box. Establish mathematical model with the energy conservation, the mass conservation theorem and the ideal gas equation of state ${ }^{[6]}$.

\section{Electronically controlled common rail fuel injection system model}

Electronically controlled common rail system is mainly composed of a high pressure oil pump, a common rail, fuel injector, fuel injection control system, various sensors and other components, which is the core of the electronic control unit, respectively realized the rail pressure regulation and fuel injection control functions ${ }^{[7]}$.

Fuel injection control

Fuel injection control system has a fuel injection control unit (WECS), injection solenoid valve, rail valve and injector parts. According to the signals from the WECS, common rail high pressure fuel is injected into the cylinder that at the optimum injection timing, injection quantity and the injection rate. The injection quantity of fuel injector fuel is function between injection duration and common rail pressure. Their relationship is showed by the following formula 


$$
\mathrm{m}=\mathrm{kt}_{i} \sqrt{p_{c}} \mathrm{I}
$$

In Eq. (3), m denotes the single cylinder fuel injection quantity; $\mathrm{k}$ denotes the injection coefficient, and is related with the solenoid valve operating characteristics, common rail pressure, nozzle structure and size; $t_{i}$ denotes the injection duration; $p_{c}$ denotes the common rail pressure.

The control model of rail pressure

According to the mass conservation theorem, the amount of fuel in the common rail pipe is determined by the amount of injection fuel supply quantity, high pressure oil pump of diesel engine and the high pressure pipe leakage quantity, so the relationship between the change in common rail pressure and these 3 parameters is the following equation

$$
\frac{d p_{c}}{d t}=E \frac{m_{p}-m-m_{\text {leak }}}{V_{c}}
$$

In Eq. (4), $m_{p}$ denotes the cycle of oil production of high-pressure oil pump, In this paper, the establishment of high pressure fuel supply MAP diagram is based on the experimental data pump test bench and got the circulation oil supply of pump by look-up table with diesel engine rotation speed and quantity of the rail pressure control as parameters; $m$ denotes fuel cycle injection quantity; $m_{\text {leak }}$ denotes common rail fuel leakage, the experience value is $10 \%$ of the amount of oil; E denotes fuel volume modulus of elasticity; $V_{c}$ the high pressure oil pipe volume.

\section{The cylinder of the diesel engine}

The excess air coefficient

The excess air coefficient is an important factor affecting the diesel engine combustion and emissions and that is ratio between the actual intake amount of fuel into a cylinder and the amount of theory air that the fuel required for combustion per cycle flow into the cylinder.

Among them, the actual amount of air flowing into the cylinders (average flow) is the following equation

$$
n \oint_{i m}=\eta_{v} \frac{p_{i m} V_{d} Z_{c y l} n_{e}}{60 N_{s t} R T_{i m}}
$$

In Eq. (5), $\eta_{v}$ denotes the cylinder volume coefficient; cylinder volume; $p_{i m}$ denotes the intake pipe pressure; $Z_{c y l}$ denotes the number of cylinders; $V_{d}$ denotes the cylinder per cycle emptying volume; $n_{e}$ denotes the rotation speed of the diesel engine; $N_{s t}$ denotes the number of stroke, Two stroke is 1 , four stroke is $2 ; R$ denotes the air gas constant; $T_{i m}$ denotes the inlet temperature.

When the fuel injection quantity is $m \mathrm{~kg}$ per cycle per cylinder, then the average quality per cycle fuel into the cylinder is the following equation

$$
n \text { \& }=\frac{m N_{c y} n_{e}}{60 N_{s t}}
$$

The excess air coefficient can be obtained by (5) and (6)

$$
\alpha=\frac{n \alpha_{i n}}{n \alpha_{j} L_{o}}
$$


In Eq. (7), $L_{o}$ denotes the minimum air quality when the complete combustion of one kilogram of fuel, the general is 14.3 .

The exhaust gas temperature and torque

According to the first law of thermodynamics, the cylinder exhaust temperature can be simplified as the function of excess air coefficient.

$$
T_{e}=T_{i m}+\frac{K}{1+L_{o} \alpha}
$$

In Eq. (8), $T_{i m}$ denotes the inlet temperature; $K=\left(1-\eta_{i}-\eta_{w}\right) H_{u} / c_{p e} ; \eta_{w}$ denotes the loss of cooling; $c_{p e}$ denotes the exhaust of specific heat capacity at constant pressure.

Due to the cylinder combustion process is very complex, average model is worth to torque by the instructions. Average indicated torque is given by the following formula.

$$
T_{i}=\frac{30}{\pi} \frac{P_{i}}{n_{e}}=\frac{30}{\pi} \frac{10^{3} \eta_{i} H_{u} n}{n_{e}}
$$

where $P_{i}$ denotes the cylinder pressure indication; $\eta_{i}$ denotes the indicated thermal efficiency that is a function of the excess air coefficient, $\eta_{i}=f(\alpha) ; H_{u}$ denotes the fuel of low calorific value.

According to Hans Terkelsen experiments, the diesel engine friction loss of pressure $p_{f}$ can curve fitting as a function of engine speed.

$$
p_{f}=k_{1} n_{e}+k_{2} n_{e}^{2}
$$

where $k_{1}, k_{2}$ denotes the fitting function coefficient fitting function.

Thus the average friction torque is the following formula.

$$
T_{f}=\frac{10^{3} V_{d} p_{f}}{2 \pi N_{s t}}
$$

\section{The exhaust pipe}

The volumetric method model is used in exhaust model and regarded it as a container without regarded the heat loss, according to the quality and energy conservation and the state equation of ideal gas, exhaust pressure change can be expressed as the following formula.

$$
\hat{P}_{e m}=\frac{k_{e} R_{e}}{V_{e m}}\left(n \oint_{\partial u t} T_{e}-n \oint_{i} T_{e m}\right)
$$

where $k_{e}$ denotes the exhaust gas adiabatic index; $R_{e}$ denotes the exhaust gas constant; $V_{e m}$ denotes the exhaust volume; of $_{\text {out }}$ denotes the flow of gas from the cylinder into the exhaust pipe, according to mass conservation for cylinder into the sum of air and fuel quantity: $n \alpha_{0 u t}=n \alpha_{n}+n \alpha_{f}$; $T_{e}$ denotes the gas out of the cylinder temperature; $n_{t}$ denotes the turbine flow; $T_{e m}$ denotes the exhaust pipe gas temperature.

\section{Exhaust gas turbocharger}

Exhaust gas turbocharger included turbines, compressor and rotor. To calculate the accurate and fast, according to the turbocharger characteristics graph, used interpolation algorithm models. 


\section{Turbine}

The turbine is regarded as a nozzle and according to pressure check list to obtain flow coefficient and efficiency, thus the exhaust mass flow is the following formula.

$$
n=\mu_{t} F_{T A} \psi \frac{p_{e m}}{\sqrt{R T_{e m}}}
$$

where $\mu_{t}$ denotes the flow coefficient; $F_{T A}$ denotes the turbine nozzle equivalent area; $\psi$ denotes the flow function, its value is calculated as follows:

$$
\begin{aligned}
& \text { When } \pi_{t} \leq\left(\frac{k_{e}+1}{2}\right)^{\frac{k_{e}}{k_{e}-1}}, \\
& \psi=\sqrt{\frac{2 k_{e}}{k_{e}-1}\left(\frac{p_{b}}{p_{e m}}\right)^{\frac{2}{k_{e}}}\left[1-\left(\frac{p_{b}}{p_{e m}}\right)^{\frac{k_{e}-1}{k_{e}}}\right]}
\end{aligned}
$$

When $\pi_{t} \geq\left(\frac{k_{e}+1}{2}\right)^{\frac{k_{e}}{k_{e}-1}}, \psi$ up to a maximum value and no longer with the pressure ratio of the change, its value is

$$
\Psi_{\text {max }}=\left(\frac{2}{k_{e}+1}\right)^{\frac{k_{e}}{k_{e}+1}} \sqrt{\frac{2 k_{e}}{k_{e}+1}}
$$

where $k_{e}$ denotes the exhaust insulation coefficient; $\pi_{t}$ denotes the turbine expansion ratio.

Turbine torque is

$$
T_{t}=\frac{n_{\phi p} c_{p e} T_{e m} \eta_{t}}{n_{t c}}\left[1-\left(\frac{1}{\pi_{t}}\right)^{\frac{k_{e}-1}{k_{e}}}\right]
$$

where $T_{t}$ denotes the compressor drive torque; $T_{e m}$ denotes the exhaust pipe of exhaust gas temperature; $c_{p e}$ denotes the exhaust gas specific heat at constant pressure; $\eta_{t}$ denotes the efficiency of turbine.

\section{Compressor}

Compressor efficiency and flow can be obtained by look-up table with pressure ratio and speed of the rotor as parameters. The temperature of pressurized air when out of the machine is

$$
T_{t c}=T_{a}\left\{1+\frac{1}{\eta_{c}}\left[\left(\pi_{k}\right)^{\mu}-1\right]\right\}
$$

Absorb torque of compressor is

$$
T_{c}=\frac{n_{c} \alpha_{p} T_{a}}{\eta_{c} n_{t c}}\left[\left(\pi_{k}\right)^{\mu}-1\right]
$$

where $h_{c}$ denotes the quality of compressed air flow; $\eta_{c}$ denotes the efficiency of compressor; $n_{t c}$ denotes the rotor speed; $\pi_{k}$ denotes the supercharging ratio; $T_{a}$ denotes the compressor 
inlet temperature; $\mu=\frac{k-1}{k}, k$ denotes the adiabatic exponent of air; $c_{p}$ denotes the air specific heat at constant pressure.

\section{Rotor}

According to Newton's laws of motion can be obtained for the rotor dynamics model

$$
\underset{t c}{\mathbb{\alpha}_{t c}}=\frac{\eta_{m} T_{t}-T_{c}}{J_{t c}} \frac{60}{2 \pi}
$$

where $J_{t c}$ denotes the turbine rotor moment of inertia; $\eta_{m}$ denotes the mechanical efficiency of the turbocharger.

\section{Air cooler}

Air cooler efficiency is higher and can be simplified it as a throttling depressurization cooling segment. The empty air conditioning outlet pressure is

$$
p_{s}=p_{i m}+\Delta p_{s}
$$

where $\Delta p_{s}$ denotes the pressure drop of the air cooler, $\Delta p_{s}=\eta_{\gamma} \frac{\frac{n \alpha_{c}^{2}}{\rho_{c}}}{\text {. }}$.

The air outlet temperature cooler can be expressed as the following formula.

$$
T_{s}=T_{t c}-\eta_{s}\left(T_{t c}-T_{c w i}\right)
$$

where $T_{c w i}$ denotes the Intercooler coolant inlet temperature; $\eta_{s}$ denotes the he efficiency of cooling.

\section{Model simulation analysis}

Marine electronically controlled diesel engine propulsion system simulation model is taking a shipping company multipurpose heavy lift ship built as the parent type.

\section{Propulsion system simulation model}

System simulation model is established by Matlab/simulink software, with the $\mathrm{M}$ file storage propulsion system boundary conditions and the main parameters, Where traditional diesel models governor is replaced by high pressure common rail, high pressure oil pump and fuel injector timing control are done by WECS.

\section{The results of simulation and analysis}

On the basis of the model validation, the initial set of diesel engine working conditions for $100 \%$ load, $114 \mathrm{r} / \mathrm{min}, 80$ seconds for $50 \%$ load dump, $90.5 \mathrm{r} / \mathrm{min}$ process, 200 s added to $90 \%$ load, $110 \mathrm{r} / \mathrm{min}$, dynamic simulation experiment is conducted, research variable condition propulsion system response characteristic and ship, the diesel engine and propeller interaction mechanism, the simulation results were shown in figure 2. 

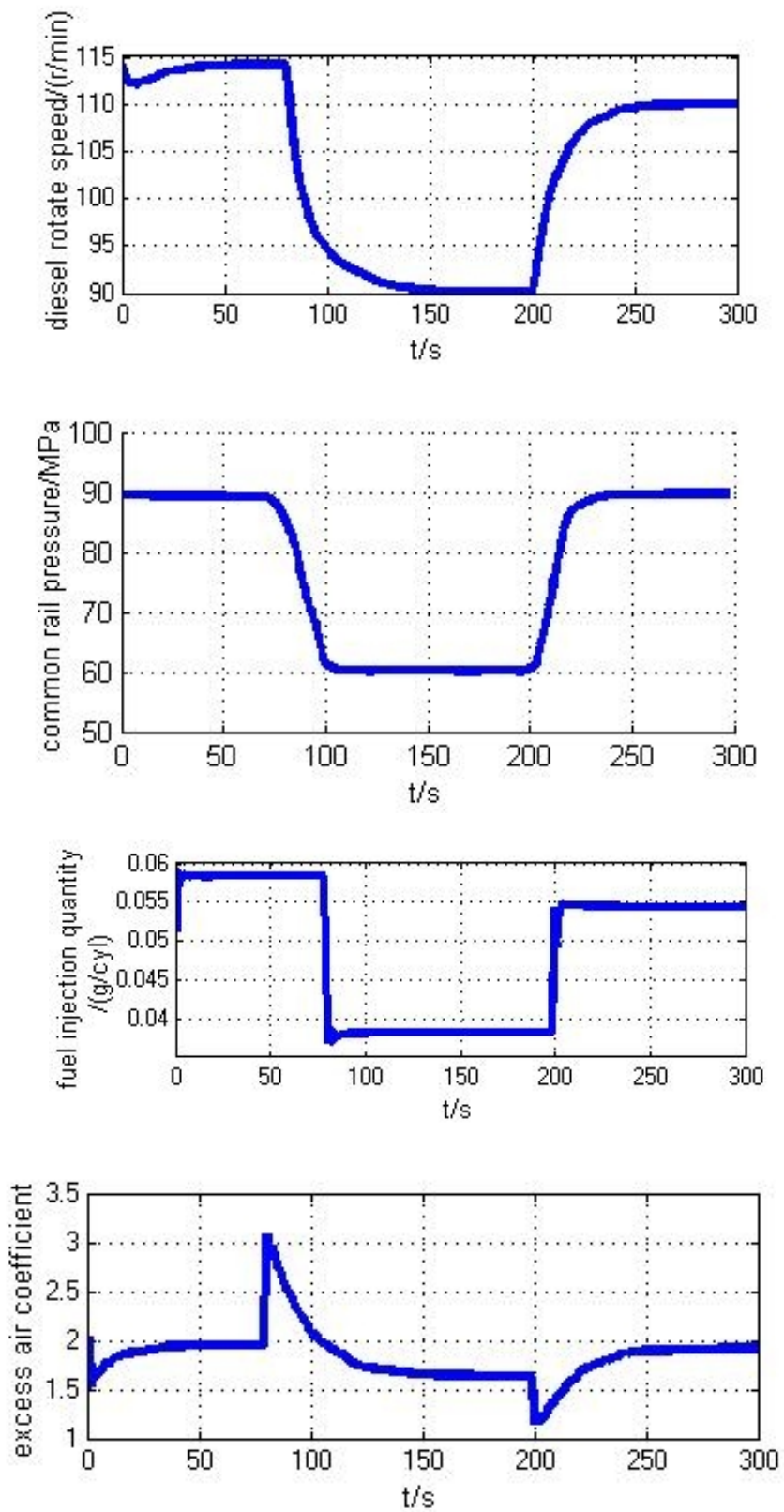

Fig.2. Propulsion system simulation model

\section{Conclusions}

This paper takes a multipurpose heavy lift vessel as the parent type, according to the characteristics of electronically controlled diesel engine, improved on the basis of traditional average values diesel engine model, by using fuzzy control strategy, rail pressure control is relatively stable, realize the control of fuel injection; diesel engine cylinder model considering the influence of excess air coefficient on the diesel engine, the mean value model is more accurate; Modeled on the propeller and hull motion, thus completing the marine electronically controlled diesel engine propulsion system model; Propulsion system as a whole model can be steady state and dynamic simulation experiment and the experimental results show that the model is accurate, and can be used as an analysis of electronic control diesel engine propulsion system features and design adaptive speed control simulation platform of the ship. 


\section{References}

[1] Huan Tu, Hui Chen. Modeling and Simulation of a Large Marine Diesel Propulsion System[J]. China shipbuilding, 2014,55 (2) :158-167.

[2] Jianbo Sun, Heng Wu, Chen Guo. Research on Modeling and Simulation of Large Marine Propulsion Plant [J]. Journal of Dalian Maritime University, 2000, 26(1):17-19.

[3] KARLSEN A T . On Modeling of a Ship Propulsion System for Control Purposes[D]. Norway Norwegian University of Science and Technology ,2012.

[4] Zheng Wang, Jianguo Yang, Yonghua Yu. Technical Analysis of the RT-flex Low-speed Intelligent Diesel Engine [J]. Marine engineering, 2009, 38 ( 5) : 100-102.

[5] Yong Chang, Yihuai Hu, Shanneng Chen. Modeling and Simulation of a CPP Propulsion System [J]. China shipbuilding, 2009, 50(2): 46-55。

[6] Haiyan Wang, Wei Zhang, Haitao Zhou. Volumetric Model of a Large-Scale Low-Speed Electronically Controlled Marine Diesel Engine [J]. Journal of internal combustion engine, 2008 (5): 452-456。

[7] Enzhe Song, Bailing Song, Xiuzhen Ma. Development of a semi-physical simulation platform for a marine diesel electronic control system [J]. Journal of Harbin engineering University, 2010, 31(9) : 1153-1160. 